Enteral tube feeding of head and neck cancer patients undergoing definitive chemoradiotherapy in the Nordic Countries : Survey of the Scandinavian Society for Head and Neck Oncology

\title{
Ilmarinen, Taru
}

2021-09

Ilmarinen , T, Hammarstedt-Nordenvall , L, Bäck , L \& Mäkitie , A 2021 , ' Enteral tube feeding of head and neck cancer patients undergoing definitive chemoradiotherapy in the Nordic Countries : Survey of the Scandinavian Society for Head and Neck Oncology ', European Archives of Oto-Rhino-Laryngology , vol. 278 , no. 9 , pp. 3489-3496 . https://doi.org/10.1007/s00405-020

http://hdl.handle.net/10138/340113

https://doi.org/10.1007/s00405-020-06545-z

unspecified

acceptedVersion

Downloaded from Helda, University of Helsinki institutional repository.

This is an electronic reprint of the original article.

This reprint may differ from the original in pagination and typographic detail.

Please cite the original version. 
Enteral tube feeding of head and neck cancer patients undergoing definitive chemoradiotherapy in the Nordic Countries: Survey of the Scandinavian Society for Head and Neck Oncology

Taru Ilmarinen ${ }^{1}$, Lalle Hammarstedt-Nordenvall ${ }^{2}$, Leif Bäck ${ }^{1}$, Antti Mäkitie ${ }^{1,2,3}$

${ }^{1}$ Department of Otorhinolaryngology - Head and Neck Surgery, Helsinki University Hospital and University of Helsinki, Helsinki, Finland

${ }^{2}$ Division of Ear, Nose and Throat Diseases, Department of Clinical Sciences, Intervention and Technology, Karolinska Institutet and Karolinska University Hospital, Stockholm, Sweden

${ }^{3}$ Research Program in Systems Oncology, Faculty of Medicine, University of Helsinki, Helsinki, Finland

Corresponding author: Taru Ilmarinen (taru.t.ilmarinen@hus.fi) 
Abstract

Purpose: We assessed current policies and perceptions of enteral tube feeding in head and neck cancer (HNC) patients undergoing chemoradiotherapy (CRT). A web-based survey was used to compare the timing and method of tube feeding within and between the five Nordic countries, covering a population of 27 million.

Methods: From each of the 21 Nordic university hospitals, one oncologist and one otorhinolaryngology head and neck (ORL-HN) surgeon responded to a survey. The respondents were asked whether tube insertion before onset of CRT, or during CRT as required (prophylactic vs. reactive) was preferred. The use of a pretreatment nutritional screening tool, and the choice of feeding route (nasogastric vs. gastrostomy tube) were assessed. In total, we analyzed responses from 21 oncologists and 21 ORL-HN surgeons.

Results: A tendency was observed towards decreasing use of a PEG tube. Of the 21 university hospitals, only $2(10 \%)$ reported using a prophylactic PEG tube in over half of HNC patients undergoing definitive CRT. The preferred method for reactive tube feeding was by a NG tube in 14 of $21(67 \%)$, and by a PEG in 7 of 21 (33\%). In general, both oncologists and ORL-HN surgeons were content with their current policy.

Conclusions: The practices for enteral tube feeding in HNC management vary within and between the Nordic countries. We suggest that unified protocols for tube feeding should be developed for this patient population.

Keywords: Chemoradiotherapy, Head and neck cancer, Nasogastric tube, Nutrition, Percutaneous endoscopic gastrostomy 
Introduction

Chemoradiotherapy (CRT) for head and neck cancer (HNC) has several side-effects that impair nutritional intake and swallowing [1]. Enteral tube feeding is often needed during and after treatment, but no consensus exists regarding the optimal method or timing [2]. A percutaneous endoscopic gastrostomy (PEG) tube or a nasogastric (NG) tube can be inserted either before treatment onset (prophylactic) or during CRT as required (reactive). Otorhinolaryngology - head and neck (ORL-HN) surgeons and oncologists have different roles in the management of HNC patients undergoing CRT. Little data exist on how tube feeding policies differ within and between countries, and whether perceptions of the optimal methods are similar between the two specialties.

Denmark (DEN), Finland (FIN), Iceland (ICE), Norway (NOR), and Sweden (SWE) all have a publicly funded healthcare system. In all Nordic countries, cancer treatment is mainly centralized to the university hospitals. Scandinavian Society for Head and Neck Oncology (SSHNO) is a multidisciplinary society aimed at unifying HNC management guidelines and promoting networking between professionals working in the field of HNC. Current policies and perceptions of tube feeding during CRT of HNC should be assessed, to provide a basis for the development and implementation of uniform guidelines. We used a web-based survey to compare the current policies and views on enteral tube feeding within and between the five Nordic countries, covering a population of 27 million.

Methods

An email with a cover letter, outlining the background and objectives of the survey, was sent to one ORL HN surgeon, and one oncologist at each Nordic university hospital treating HNC. These included four university hospitals in Denmark (Aalborg, Aarhus, Copenhagen, Odense), five in Finland (Helsinki, Kuopio, Oulu, Tampere, Turku), one in Iceland (Reykjavik), four in Norway (Bergen, Oslo, Troms $\varnothing$, Trondheim), and seven in Sweden (Göteborg, Linköping, Lund, Stockholm, Umeå, Uppsala, Örebro). From each center, one oncologist and one ORL-HN surgeon were asked to participate, using a link provided in an email. The survey 
was not limited to a specific oncology subspecialty, both medical and radiation oncologists were accepted as respondents. A reminder email was sent in March, and a 100\% response rate was obtained in April 2020. The survey was completed anonymously, although the participants were asked to report their department, specialty (oncology or ORL-HN surgery), city, and country. The survey included four structured questions concerning the current policy for pre-treatment nutritional assessment and enteral tube feeding, followed by four questions inquiring the respondent's personal expert opinion on tube feeding and on two patient cases. After each structured question, a space was provided for free comments.

In some cases, the views on current policies differed slightly between two specialists within the same university hospital. In these cases, a consensus was made by the authors based on the participants' free comments.

Statistical analyses

The data are presented as percentage of university centers, or percentage of respondents from each country. The data on professional expert opinion are also presented by specialty. IBM SPSS Statistics 25.0 was used for statistical analyses. Chi-square and Fisher's tests were used to compare responses from ORL - HN surgeons and oncologists.

Results

All 21 university hospitals from the five Nordic countries participated in the study. Thus, data were analyzed from 42 respondents, including 21 oncologists and 21 ORL-HN surgeons.

Current policies: The use of a validated screening tool to assess nutritional status 
The first question involved the use of a validated screening tool to assess nutritional status before treatment onset. The respondents were also asked to specify which tool they used. The use of nutritional screening tools in each country is presented in Fig. 1 . Of 21 centers, $13(62 \%)$ reported that they were either encouraged to use a screening tool or used it routinely. Of 21 centers, only 7 (33\%) reported using Nutritional Risk Screening 2002 (NRS2002). Some reported using a validated screening tool but were unable to specify the specific method because another professional was responsible for it. According to the free comments, the oncologist did the screening in some centers, while in others the ORL-HN surgeon or a specialist dietitian was responsible. Although the question specifically involved the use of a validated tool, some centers only evaluated reduction in weight or body mass index (BMI), or used a hospital specific method.

Current policies: The use of a prophylactic percutaneous endoscopic gastrostomy (PEG) tube

The next question involved the use of a prophylactic percutaneous endoscopic gastrostomy (PEG) tube for HNC patients undergoing CRT (Fig. 2a). The respondents were also asked to specify whether prophylactic PEG was applied in over half of patients undergoing definite CRT. In 16 of 21 (76\%) centers, prophylactic PEG placement was routinely considered before onset of CRT. Only two centers (one in Denmark, one in Finland) reported that a prophylactic PEG was applied in over half of all patients undergoing definite CRT. Contrarily, in 5 of 21 (24\%) centers a prophylactic PEG was never applied if the patient can swallow before onset of CRT.

Current policies: The decision of a prophylactic PEG

The participants were asked whether the decision of prophylactic PEG insertion was made by the multidisciplinary tumor board (MTB) meeting, by the oncologist, or by the ORL-HN surgeon. Fig. $2 \mathrm{~b}$ presents the responses from each country. In 10 of 21 (48\%) centers, the decision was made by the MTB meeting. Of 21 centers, 7 (33\%) either had no protocol for prophylactic PEG decision, or never considered a prophylactic 
PEG. In centers without a fixed protocol for prophylactic PEG decision, the recommendation was given either by the MTB meeting or the treating physician.

Current policies: The method for reactive tube feeding

According to the data above, the policy for tube feeding in most centers was reactive rather than prophylactic. Thus, enteral tube feeding during CRT was considered only if the patient was unable to maintain normal swallowing. In 14 of 21 (67\%) centers, the preferred method for reactive tube feeding was by a NG tube. However, in all five (100\%) Finnish centers, and in two of four (50\%) Danish centers, the preferred method for reactive tube feeding was PEG. The specific question and responses from each country are presented in Fig. 3. In cases of discrepancy between two respondents within the same center, the free comments were assessed to make a consensus. Some respondents remarked that the decision will depend on the anticipated length of tube feeding, and on patient preference. Some also commented that the decision of reactive tube feeding was by another specialty.

Expert opinion on the use of a prophylactic PEG

The next section inquired personal expert opinion on whether the use of a prophylactic PEG should increase, remain as it is, or increase. Most respondents were satisfied with their current policy; 29 of 42 (69\%) considered that the use of a prophylactic PEG should remain as it is. Of the 11 respondents calling for a change in the current policy for prophylactic PEG use, five of 11 hoped for an increase, and 6 of 11 for a decrease. There were no statistically significant differences in the responses from ORL-HN surgeons and oncologists ( $p=0.66)$. Fig. 4 presents the data from each country, and by the respondents' specialty. The free comments from several centers revealed that the use of a prophylactic PEG had decreased. It was considered only when significant weight loss was observed prior to treatment, in hypopharyngeal cancer, in palliative patients, and when swallowing difficulties were expected to last over 8 weeks. Several respondents also 
stated that short-term use of NG tube was typically easy and feasible. Many called for a uniform guideline to support decision making for enteral tube feeding.

Expert opinion on the effect of prophylactic PEG on unplanned hospital admissions

We asked whether the respondents perceived an association between the use of a prophylactic PEG and unplanned hospital admissions. Fig. 5 presents the data from each country, and by respondents' specialty. The impression that unplanned hospital admissions are more common in patients without a prophylactic PEG was more common among oncologists (10 of $21,48 \%$ ) than ORL-HN surgeons ( 7 of $21,33 \%$ ). However, the differences between the two specialties were not statistically significant $(p=0.35)$. The greatest proportion of respondents agreeing that the lack of prophylactic PEG impacts on unplanned hospital admissions were from Finland (6 of 10) and from Sweden (6 of 12), although the tendency for prophylactic and reactive PEG insertion was high in Finland, but low in Sweden. In Norway, with a low tendency for both prophylactic and reactive PEG insertion, only 1 of 8 agreed. Of 21 respondents, 9 (43\%) either had no opinion or were unable to answer because prophylactic PEG was not their policy.

Expert opinion on tube feeding in oropharyngeal and hypopharyngeal cancer

To assess the preferred policy for tube feeding more specifically, we presented two patient cases. For a 55year-old patient with p16 positive base of tongue squamous cell carcinoma, 23 of $42(55 \%)$ respondents preferred a reactive NG tube. For a 65-year-old patient with hypopharyngeal squamous cell carcinoma, 28 of 42 (67\%) respondents preferred a prophylactic PEG. The exact questions and responses from each country are presented in Fig. $6 \mathrm{a}$ and $6 \mathrm{~b}$. There were no statistically significant differences in the perceptions of ORL$H N$ surgeons and oncologists ( $p=0.20$ and $p=0.73$, respectively).

Discussion 
Evaluation of nutritional status before onset of CRT

Our survey showed that in the Nordic countries, pre-treatment evaluation of nutritional status is variable. In most centers, the decision of prophylactic tube feeding was either made by an oncologist or by the MTB meeting. The exact method for nutritional screening, however, was often unclear. In HNC patients, weight loss both before and during radiotherapy is an important prognostic indicator for disease specific survival. Critical weight loss of $5 \%$ or more during treatment has been associated with worse survival outcomes [3]. Thus, evaluation of nutritional status before, during, and after CRT is crucial. Pre-treatment evaluation of comorbidities and nutritional status, using validated tools, should be a part of the standard management of HNC patients. In the Nordic countries, the tools for evaluation should preferably be similar, allowing comparison and combination of data when planning and implementing new protocols.

\section{Prophylactic vs. reactive tube feeding}

In our survey, several respondents stated that enteral tube feeding is applied during CRT only if necessary. NG tube insertion without CRT interruption appeared safe and easy, and stood out as the preferred method for reactive tube feeding. In oropharyngeal cancer, the need for long-term tube feeding may decline in the future by less toxic, de-escalated treatment protocols [4]. The number of unplanned hospital admissions should be considered in prospective studies comparing prophylactic vs. reactive tube feeding policies. Ten out of the 21 oncologists in our survey agreed that the lack of prophylactic PEG was associated with unplanned hospital admissions. With a reactive tube feeding policy, repeated evaluation of nutritional status and swallowing remains important.

There is no consensus for the optimal timing of tube insertion. A recent review study showed that a prophylactic PEG insertion reduced the number of malnourished patients (defined as $>10 \%$ of body weight), but average weight loss at various time points following treatment between prophylactic and reactive PEG groups was similar. The percentage of patients in the reactive group eventually ending up with PEG in different studies ranged considerably, from 12 to $73 \%$ [5]. 
There are concerns that a prophylactic PEG may negatively affect swallowing physiology and function. However, the evidence to support the association between prophylactic PEG and long-term tube dependence is weak $[6,7]$. The value of retrospective studies is limited since the prophylactic and reactive groups typically have significant baseline differences.

United Kingdom national multidisciplinary guidelines recommend enteral nutrition if the anticipated food intake (60 per cent of estimated energy expenditure) is decreased for more than 10 days, and that gastrostomy insertion should be considered when tube feeding is anticipated for more than four weeks [8] . However, there are no validated tools available for predicting which patients exactly are at risk for prolonged tube feeding. According to the previous literature, the critical parameters include age, pretreatment weight loss, comorbidities, tumor stage and subsite, as well as radiation dose and distribution [9]. In our survey, prophylactic PEG was considered justified when significant weight loss was observed prior to treatment, in hypopharyngeal cancer, in palliative patients, and when prolonged dysphagia was anticipated.

Percutaneous endoscopic gastrostomy (PEG) vs. nasogastric (NG) tube

Our survey revealed a shift towards using NG tube rather than PEG. The choice of preferred feeding route (PEG vs. NG) presumably depends upon local arrangements. A Finnish study showed that PEG insertion by ORL-HN surgeons was feasible and safe, and major complications occurred only in $3.2 \%$. Compared to the previous era when gastrointestinal surgeons inserted the PEG tubes, the delay for PEG insertion by ORL-HN surgeons was shorter [10]. In our survey, two centers reported that severe PEG-related complications had led to the reduced use of PEG. However, in some countries, financial compensation for nutritional supplies is only granted for patients with PEG, which might be a contributing factor.

Patient preferences should also be considered in the choice of feeding route. A recent study from the UK assessed the feasibility of feeding routes in a randomized controlled trial, comparing oral feeding plus pretreatment gastrostomy with oral feeding plus as-required NG tube feeding in patients with HNC. The trial was closed following poor recruitment; only 17 of 75 (23\%) patients consented to be randomized. Neither 
method was especially preferred by the majority of patients [11]. Important aspects from the patients' perspective include discomfort related to tube irritation and dislodgement, social and physical functioning, adherence to swallowing exercises, and willingness to maintain a normal diet. Few studies have analyzed the association between feeding route and quality of life. Higher incidence of pain has been reported with gastrostomy tubes in the first week of insertion. Disadvantages of NGT include altered body image, and problems with cosmesis and mobility [12].

\section{Limitations}

This survey has some limitations. Some discrepancy occurred in the views of current policy between respondents even within the same university hospital. This is likely to reflect the lack of fixed guidelines at these centers. Furthermore, responses from only two participants may not be representative of all specialists within the university hospital, although awareness of common guidelines should be preferable. Our survey was not limited to a specific oncology subspecialty, partly because radiation oncology and medical oncology are integrated into disease oriented clinical oncology in some Nordic centers. All respondents were dedicated to treating head and neck cancer. Although the exact timing and method for consultation with a specialist dietitian was not specified, several respondents emphasized the role of nutritional services in treatment planning. The exact reasons for timing and method of tube feeding was not asked by using structured questions.

\section{Conclusions}

The practices for enteral tube feeding vary within and between the Nordic countries. Nasogastric tube remains the preferred method for reactive tube feeding in most centers. Our survey found a tendency towards decreased use of a prophylactic percutaneous endoscopic gastrostomy tube. Although a decrease in the use of PEG was observed both in prophylactic and in reactive setting, it still has a role in selected patients. 
In our survey, participants from centers with a strong tendency for PEG use were content with the current situation, but so were participants from centers preferring NG tube. Professional expert opinions on two patient cases showed that there were supporters for all existing methods of tube feeding. This may indicate that they all truly have certain benefits over another. In a setting where all centers consider their method to be the most feasible one, implementing a common protocol may be challenging.

We suggest that unified protocols for tube feeding should be developed for this patient population. Nutritional status should be assessed using a validated screening tool, and a specialist dietitian should be a part of the multidisciplinary team. Insertion of a feeding tube before treatment onset should be considered in patients with malnutrition at baseline. Patients who are at risk for malnutrition (NRS2002 score $\geq 3$ ) should receive counselling from a specialist dietitian. Weight and nutritional intake should be monitored weekly during CRT, and on each post-treatment follow-up visit.

In patients without an obvious risk for malnutrition at baseline, NG tube insertion when needed during CRT could be the primary option. Both in prophylactic and in reactive setting, however, individual patient preferences should be considered in the decision between NG tube and PEG. Transition from NG tube to PEG should be regarded as an alternative, in case swallowing difficulties continue. Patient satisfaction, and the effect of tube feeding method on quality of life parameters, should be measured to guide decision making in the future.

Figure Captions

Fig. 1 Responses to the question "When definitive chemoradiotherapy (CRT) is planned for a patient with HNC, do you use a validated screening tool to assess nutritional status before treatment onset?" The X-axis presents the percentage of university centers. 
Fig. 2a Responses to the question "When definitive chemoradiotherapy (CRT) is planned for a patient with HNC, do you routinely consider prophylactic percutaneous endoscopic gastrostomy (PEG) tube placement in your institution before treatment onset?" The X-axis presents the percentage of university centers.

Fig. $2 b$ Responses to the question "When definitive chemoradiotherapy (CRT) is planned for a patient with HNC, who decides whether prophylactic PEG placement is recommended before treatment onset?" The Xaxis presents the percentage of university centers. In centers with no protocol, the decision was made either by the multidisciplinary tumor board meeting or the treating physician, or the question was irrelevant since a prophylactic PEG was never applied.

Fig. 3 Responses to the question: "A HNC patient is undergoing CRT. Four weeks after treatment onset, the patient has difficulties in swallowing. After instructions from a speech-language therapist, adequate pain medication, and intravenous fluid administration at the hospital ward, the patient is still unable to restore normal swallowing. In your institution, what is the preferred way to proceed?" The X-axis presents the percentage of university centers.

Fig. 4 Responses to the question: "YOUR PERSONAL EXPERT OPINION: The use of prophylactic PEG in patients undergoing CRT should a) increase b) remain as it is c) decrease." The X-axis presents the percentage of respondents.

Fig. 5 Responses to the question: "YOUR PERSONAL EXPERT OPINION: Unplanned hospital admissions during CRT are more common in patients without a prophylactic PEG, compared to those with a prophylactic PEG" The $\mathrm{X}$-axis presents the percentage of respondents.

Fig. 6a Responses to the question: "YOUR PERSONAL EXPERT OPINION: 55-year old male without significant medical history is diagnosed with p16-positive base of tongue squamous cell carcinoma (T2N1M0). Body mass index (BMI) is 25 , without significant weight loss, pain, or difficulty in swallowing. MTB meeting recommends definitive CRT. In your opinion, what is the best tube feeding strategy?" 
Fig. 6b Responses to the question: "YOUR EXPERT OPINION: 65-year old male with a long history of tobacco and alcohol abuse is diagnosed with a hypopharyngeal squamous cell carcinoma, affecting the postcricoid area (T2N2bM0). The patient reports difficulties in swallowing for 2 months, with irregular use of pain medication. BMI is 19, with 5\% weight loss in 2 months. If MTB meeting recommends definitive CRT what, in your opinion, is the best tube feeding strategy?"

Declarations

Funding: The author(s) received no financial support for the research

Conflicts of interest: The authors declare that they have no conflict of interest

Ethics approval: Not applicable

References

1. Kristensen MB, Isenring E, Brown B (2020) Nutrition and swallowing therapy strategies for patients with head and neck cancer. Nutrition 69: 110548. doi: 10.1016/j.nut.2019.06.028. Epub 2019 Jul 2

2. Brown TE, Banks MD, Hughes BGM, Lin CY, Kenny LM, Bauer JD (2017) Randomised controlled trial of early prophylactic feeding vs standard care in patients with head and neck cancer. Br J Cancer 117:15-24

3. Langius JA, Bakker S, Rietveld DH, Kruizenga HM, Langendijk JA, Weijs PJ, Leemans CR (2013) Critical weight loss is a major prognostic indicator for disease-specific survival in patients with head and neck cancer receiving radiotherapy. $\mathrm{Br} J$ Cancer 109:1093-1099

4. Foster CC, Seiwert TY, MacCracken E, Blair EA, Agrawal N, Melotek JM, Portugal L, Brisson RJ, Gooi Z, Spiotto MT, Vokes EE, Haraf DJ (2020) Dose and Volume De-Escalation for Human Papillomavirus-Positive Oropharyngeal Cancer is Associated with Favorable Posttreatment Functional Outcomes. Int J Radiat Oncol Biol Phys 107: 662-671 
5. McClelland S,3rd, Andrews JZ, Chaudhry H, Teckie S, Goenka A (2018) Prophylactic versus reactive gastrostomy tube placement in advanced head and neck cancer treated with definitive chemoradiotherapy: A systematic review. Oral Oncol 87:77-81

6. Shaw SM, Flowers H, O'Sullivan B, Hope A, Liu LW, Martino R (2015) The effect of prophylactic percutaneous endoscopic gastrostomy (PEG) tube placement on swallowing and swallow-related outcomes in patients undergoing radiotherapy for head and neck cancer: a systematic review. Dysphagia 30:152-175

7. Setton J, Lee NY, Riaz N, Huang SH, Waldron J, O'Sullivan B, Zhang Z, Shi W, Rosenthal DI, Hutcheson KA, Garden AS (2015) A multi-institution pooled analysis of gastrostomy tube dependence in patients with oropharyngeal cancer treated with definitive intensity-modulated radiotherapy. Cancer 121:294-301

8. Talwar B, Donnelly R, Skelly R, Donaldson M (2016) Nutritional management in head and neck cancer: United Kingdom National Multidisciplinary Guidelines. J Laryngol Otol 130:S32-S40

9. MD Anderson Head and Neck Cancer Symptom Working Group, Spatial-Non-spatial Multi-Dimensional Analysis of Radiotherapy Treatment/Toxicity Team (SMART3) (2019) Chronic radiation-associated dysphagia in oropharyngeal cancer survivors: Towards age-adjusted dose constraints for deglutitive muscles. Clin Transl Radiat Oncol 18:16-22

10. Ruohoalho J, Aro K, Makitie AA, Atula T, Haapaniemi A, Keski-Santti H, Kylanpaa L, Takala A, Back LJ (2017) Prospective experience of percutaneous endoscopic gastrostomy tubes placed by otorhinolaryngologisthead and neck surgeons: safe and efficacious. Eur Arch Otorhinolaryngol 274:3971-3976

11. Paleri V, Patterson J, Rousseau N, Moloney E, Craig D, Tzelis D, Wilkinson N, Franks J, Hynes AM, Heaven B, Hamilton D, Guerrero-Urbano T, Donnelly R, Barclay S, Rapley T, Stocken D (2018) Gastrostomy versus nasogastric tube feeding for chemoradiation patients with head and neck cancer: the TUBE pilot RCT. Health Technol Assess 22:1-144

12. Wang J, Liu M, Liu C, Ye Y, Huang G (2014) Percutaneous endoscopic gastrostomy versus nasogastric tube feeding for patients with head and neck cancer: a systematic review. J Radiat Res 55: 559-567 
The use of a nutritional screening tool before chemoradiotherapy

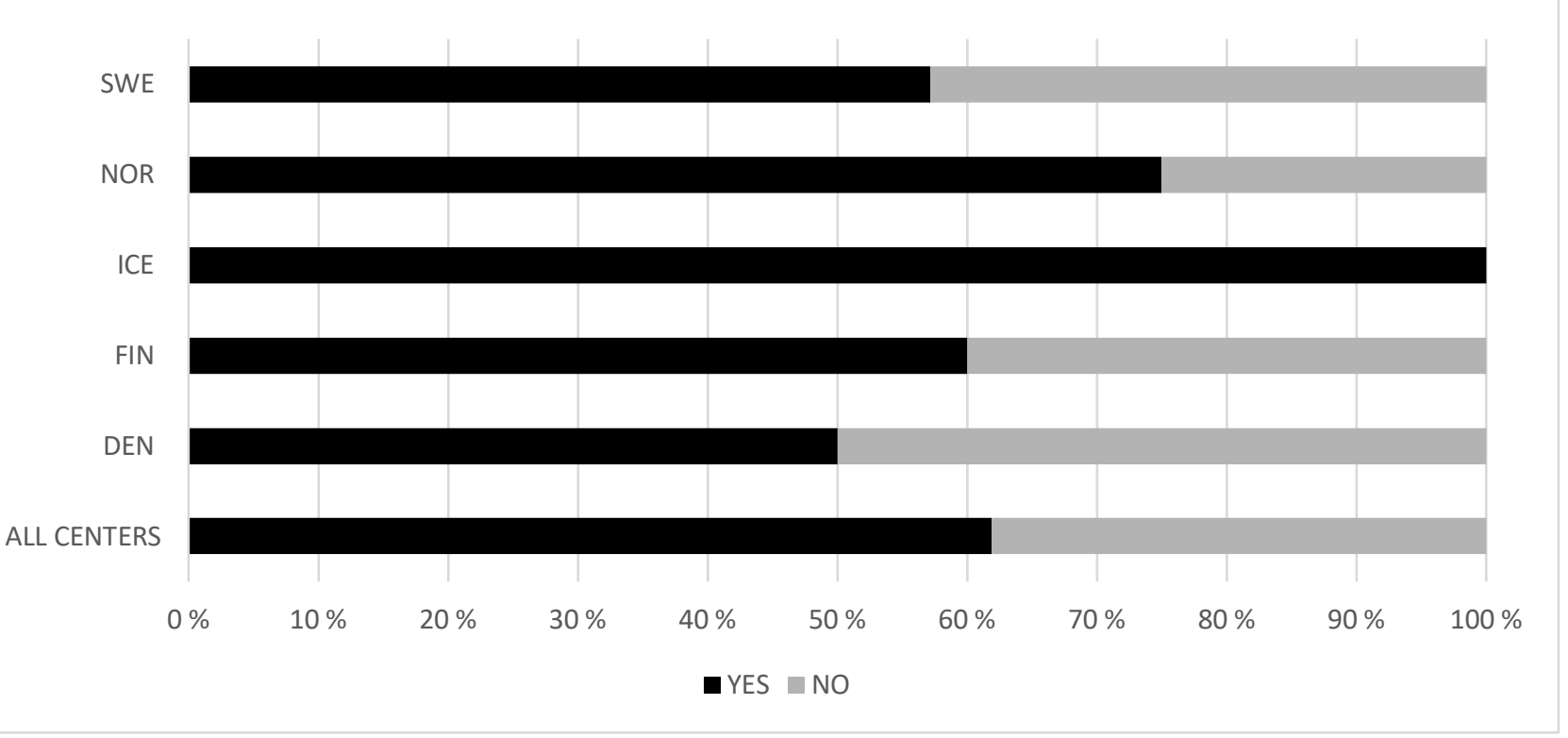

Fig 1. 
Prophylactic PEG is routinely considered before definitive chemoradiotherapy

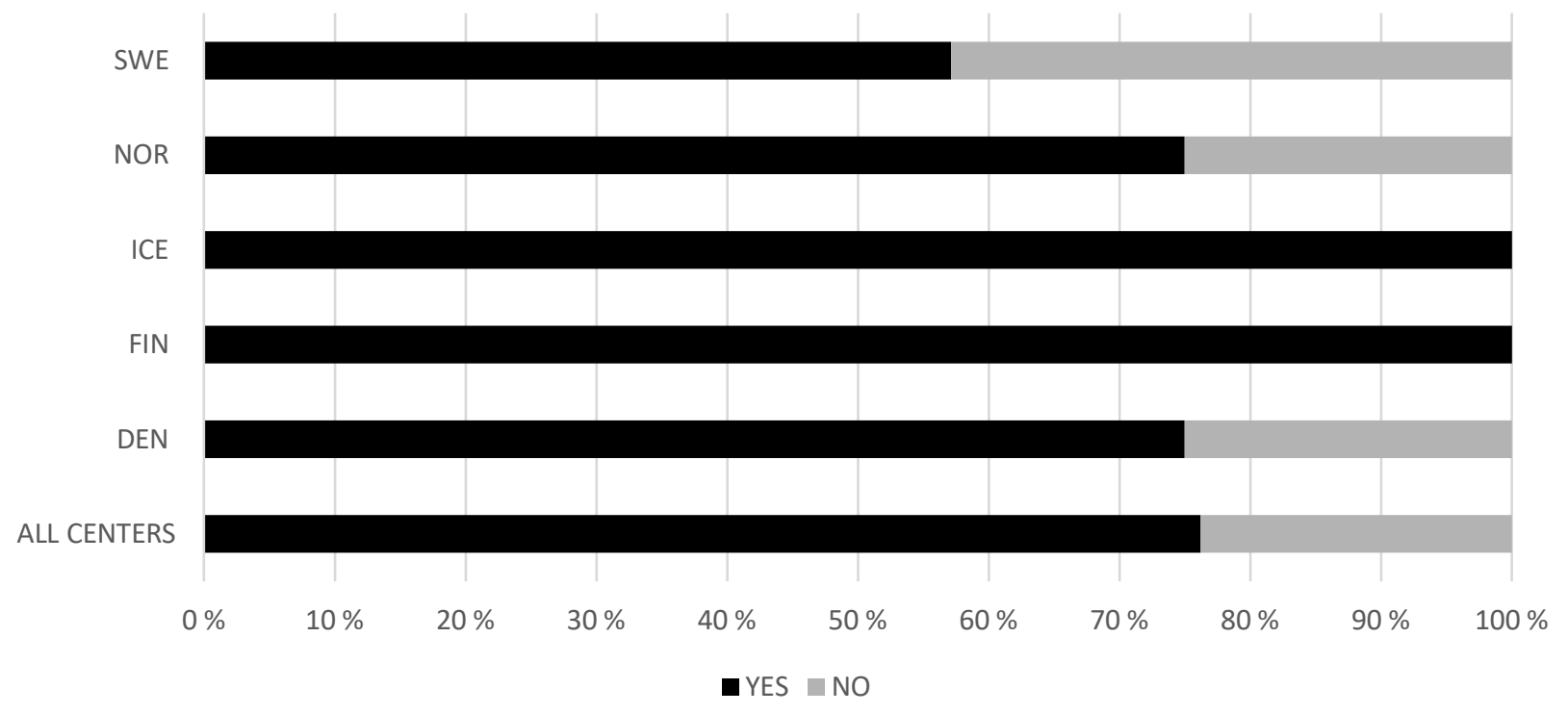

Fig. $2 a$

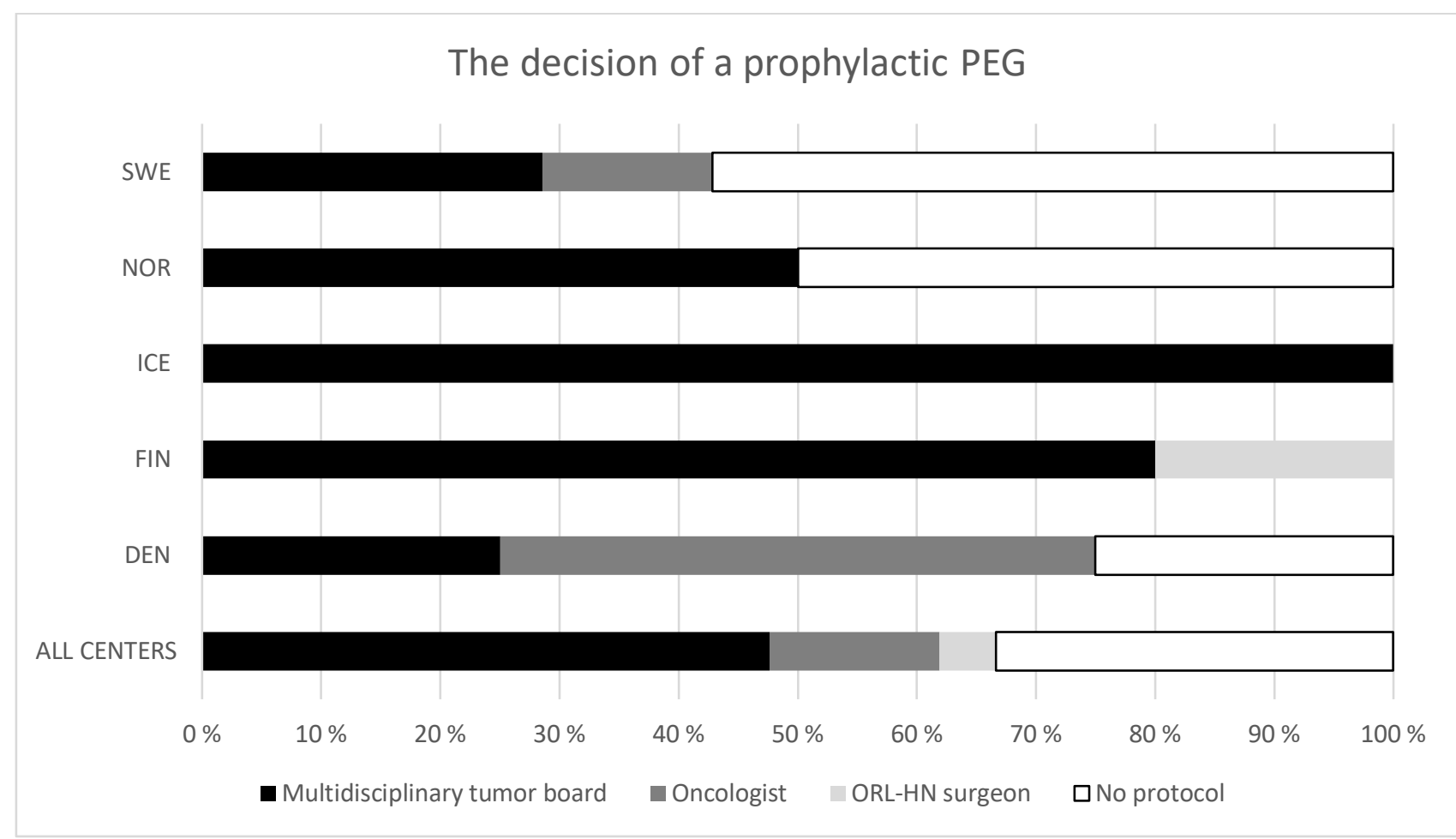

Fig. $2 b$ 


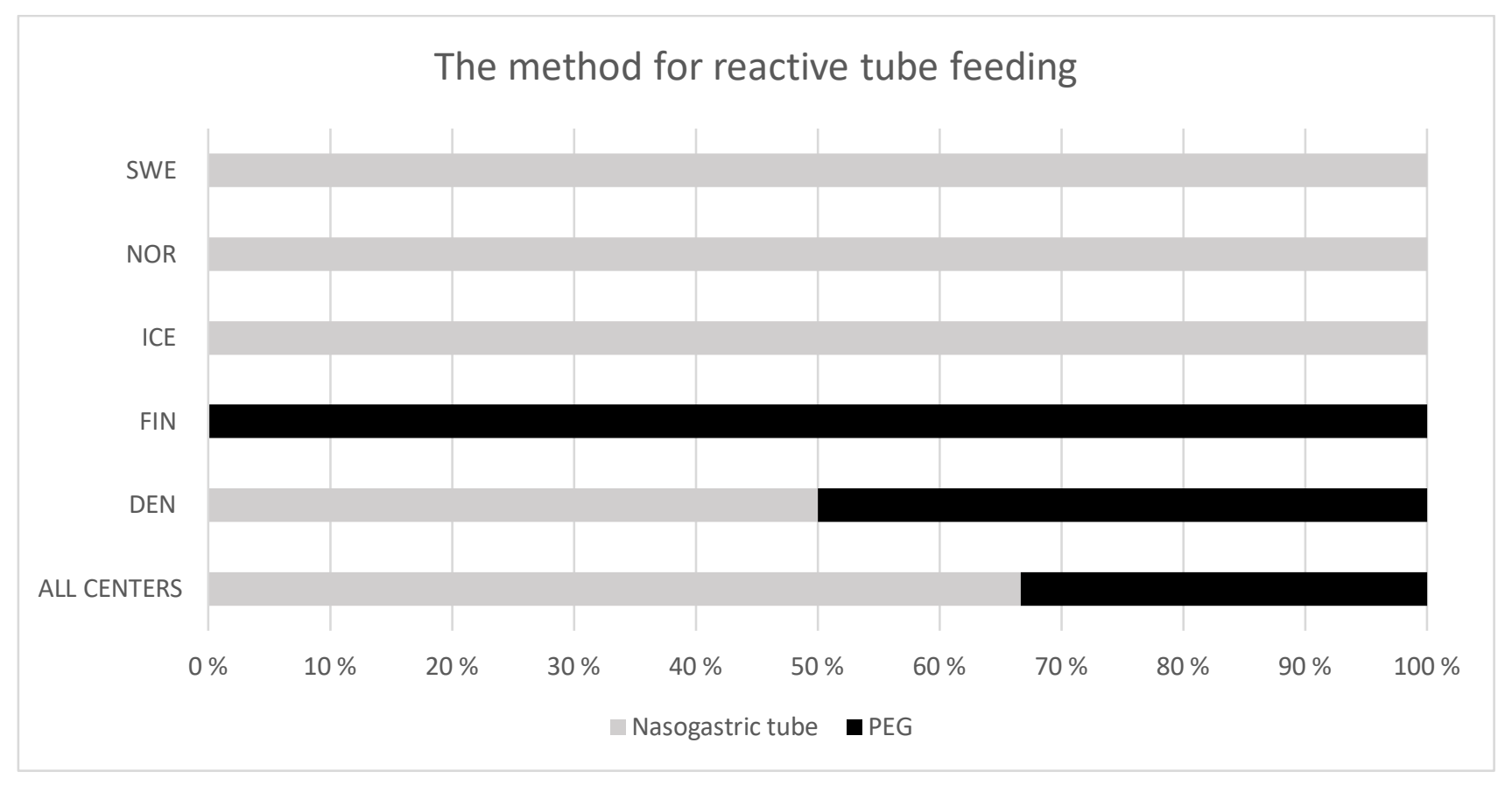

Fig. 3 


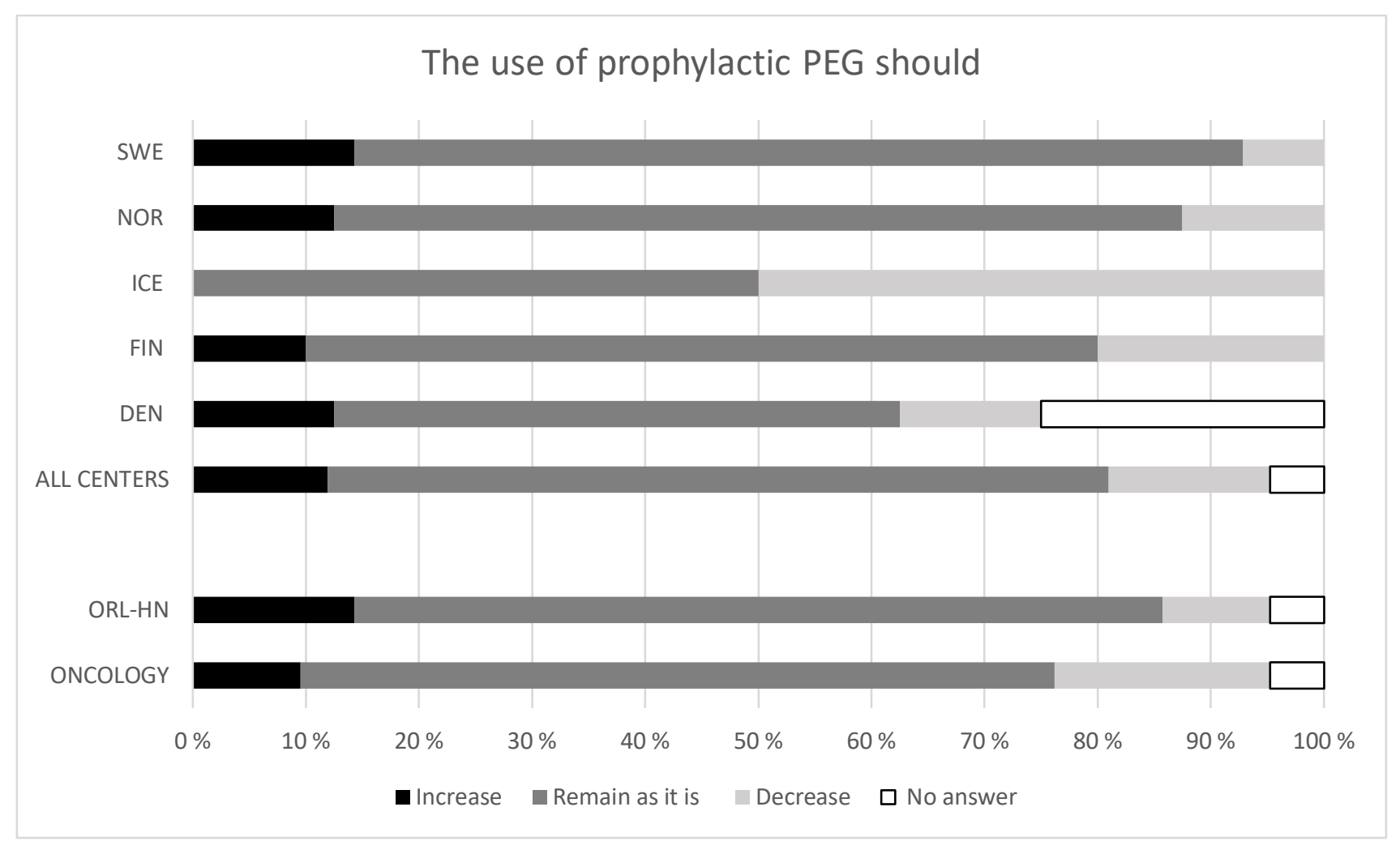

Fig. 4 


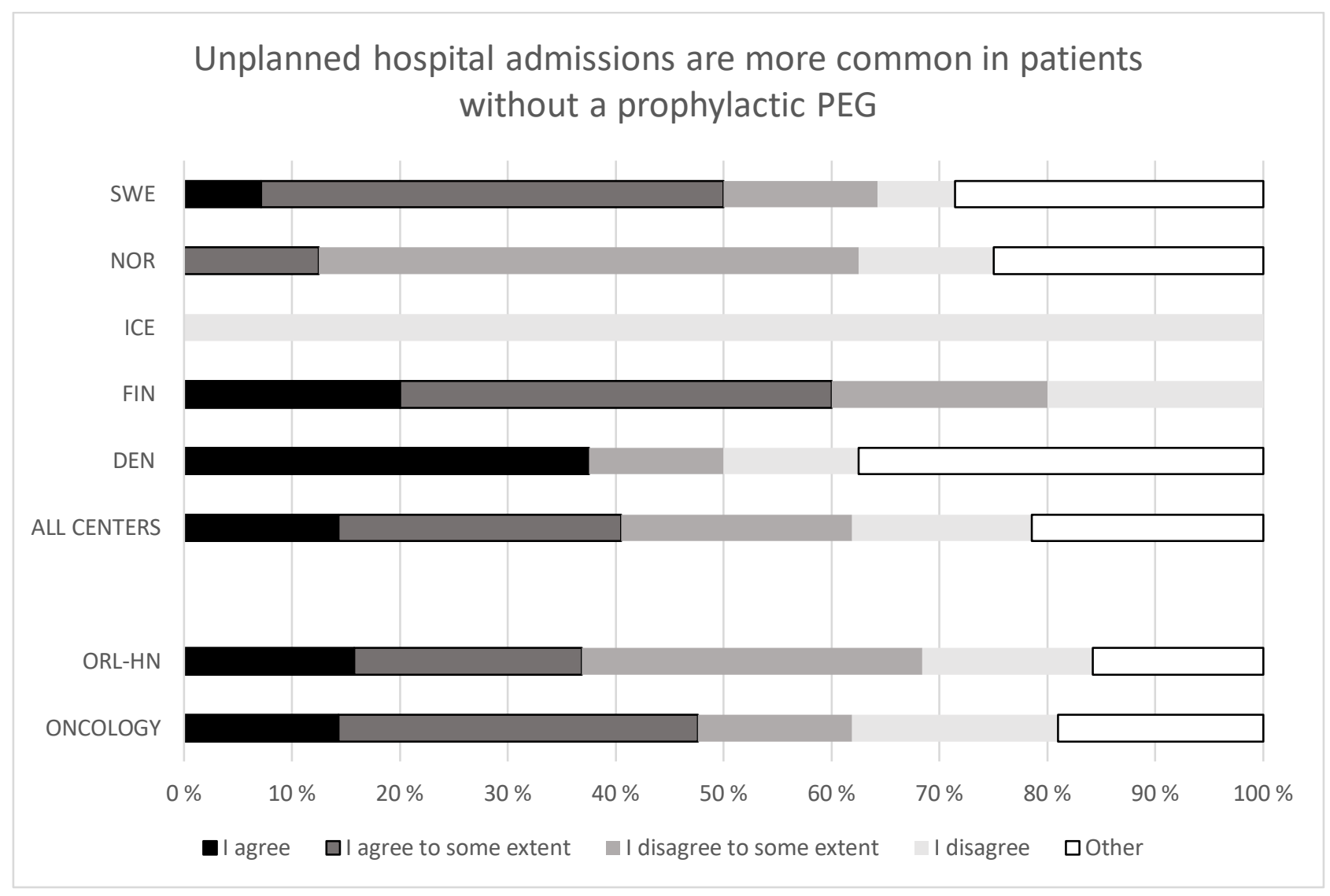

Fig. 5. 
The best tube feeding strategy in base of tongue squamous cell carcinoma

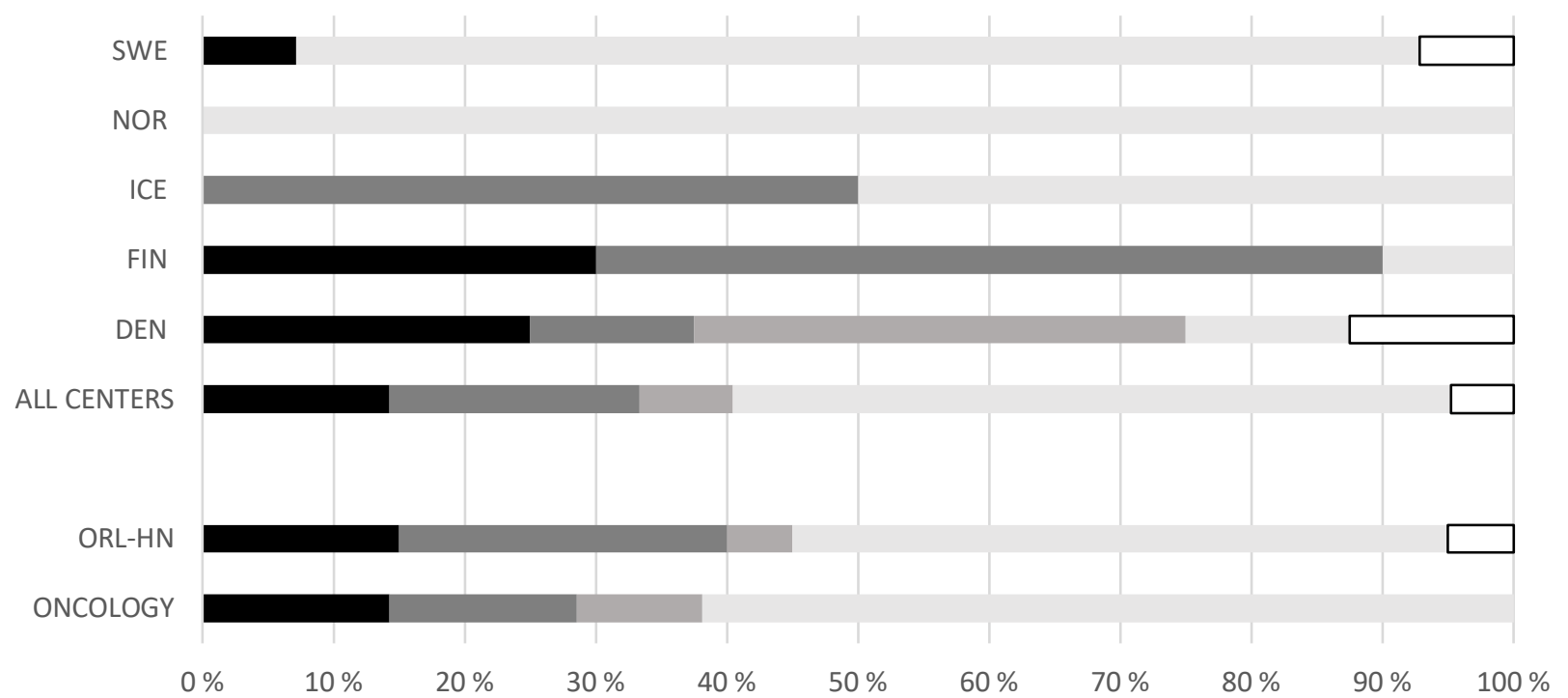

- Prophylactic PEG $\quad$ Reactive PEG $\quad$ Prophylactic nasogastric tube Reactive nasogastric tube $\square$ Other

Fig. $6 a$

The best tube feeding strategy in hypopharyngeal squamous cell carcinoma

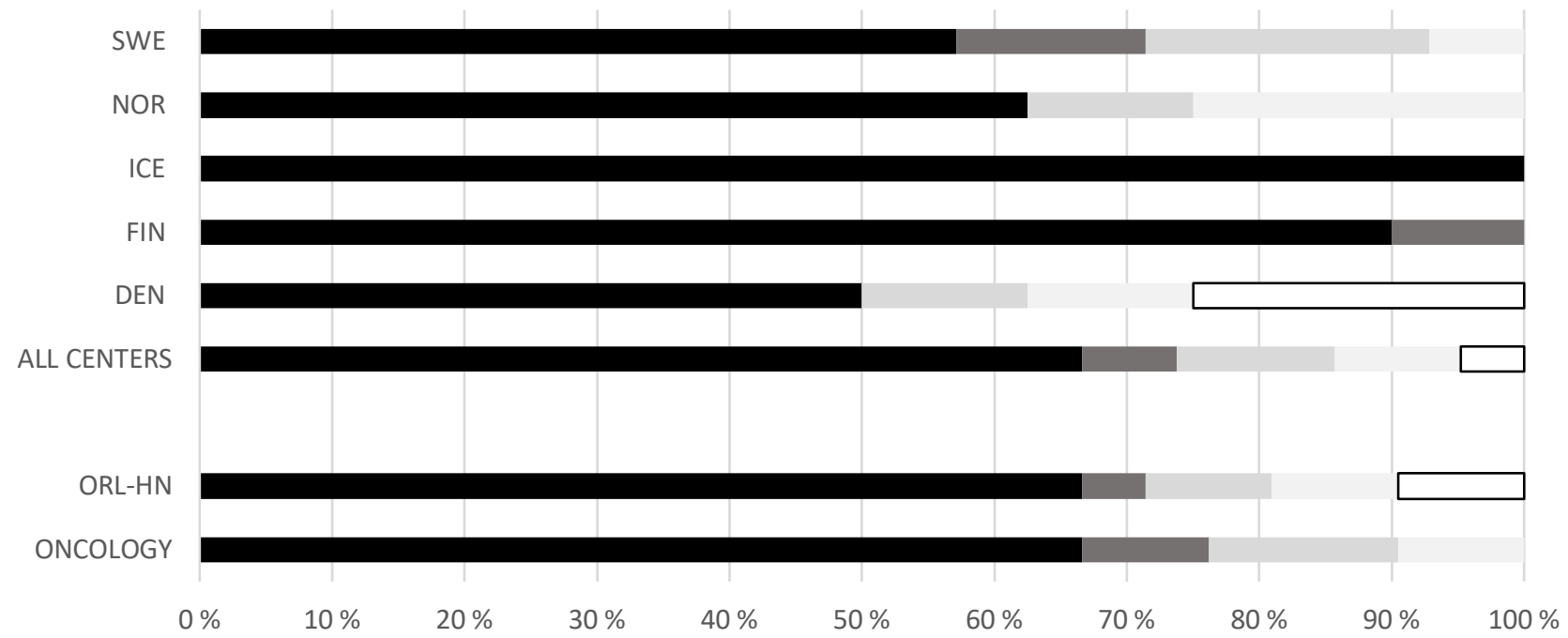

- Prophylactic PEG $\square$ Reactive PEG Prophylactic nasogastric tube Reactive nasogastric tube $\square$ Other

Fig. $6 b$ 
